Supplement of Geosci. Model Dev., 14, 1125-1145, 2021

https://doi.org/10.5194/gmd-14-1125-2021-supplement

(c) Author(s) 2021. This work is distributed under

the Creative Commons Attribution 4.0 License.

(c) (1)

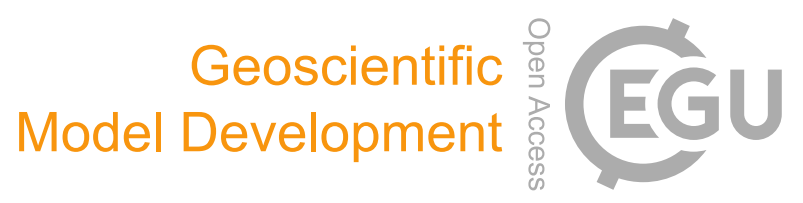

Supplement of

\title{
Global storm tide modeling with ADCIRC v55: unstructured mesh design and performance
}

William J. Pringle et al.

Correspondence to: William J. Pringle (wpringle@anl.gov)

The copyright of individual parts of the supplement might differ from the CC BY 4.0 License. 


\section{S1 ADCIRC v55 Formulation and Solution Scheme}

\section{S1.1 Reformulation of Governing Equations}

To facilitate a Continuous Galerkin Finite Element Method (CG-FEM) solution to the governing equations in spherical coordinates (Eqs. (1)-(3) from the main manuscript) with minimal modification to FEM methods that exist for Cartesian coordinates

5 we use a rectilinear mapping projection. Previously in ADCIRC, the Carte Parallelogrammatique projection (CPP) has been used (Kolar et al., 1994a), which is an equidistant cylindrical projection that is neither area preserving (equal-area) nor angle preserving (conformal). In the TELEMAC model a conformal cylindrical Mercator projection is used (Hervouet, 2007). Here, we introduce a generalized cylindrical mapping system in which equal-area, equidistant, or conformal options can be arbitrarily selected through the integer, $p$. The resulting transformation equations to map $(\lambda, \phi)$ into $(x, y)$ are,

$10 x=R\left(\lambda-\lambda_{0}\right) \cos \phi_{0}$

$y= \begin{cases}R \sin \phi \sec \phi_{0} & \text { if } p=0: \text { Equal-area } \\ R \phi & \text { if } p=1: \text { Equidistant (CPP) } \\ R \ln (\tan \phi+\sec \phi) \cos \phi_{0} & \text { if } p=2: \text { Conformal (Mercator) }\end{cases}$

where $\left(\lambda_{0}, \phi_{0}\right)$ is the arbitrary projection origin. Applying this transformation to the governing equations (Eqs. (1)-(3) from the main manuscript) results in the following form,

$$
\begin{aligned}
\frac{\partial\left(\zeta \cos ^{p} \phi\right)}{\partial t}= & -L_{x} \frac{\partial(U H)}{\partial x}-L_{y} \frac{\partial(V H \cos \phi)}{\partial y} \\
\frac{\partial U}{\partial t}= & -K U \frac{\partial U}{\partial x}-K^{p-1} V \frac{\partial U}{\partial y}-K \frac{\partial \Psi}{\partial x}-\left(\mathcal{C}_{x y}-f^{\prime}\right) V+\tau_{w} U_{w}-\left(\tau_{b}+\mathcal{C}_{x x}\right) U \\
& +\nu_{t}\left[K \frac{\partial \tau_{x x}}{\partial x}+K^{p-1} \frac{\partial \tau_{x y}}{\partial y}-\tan \phi\left(\tau_{x y}+\tau_{y x}\right)\right] \\
\frac{\partial V}{\partial t}= & -K U \frac{\partial V}{\partial x}-K^{p-1} V \frac{\partial V}{\partial y}-K^{p-1} \frac{\partial \Psi}{\partial y}-\left(\mathcal{C}_{y x}+f^{\prime}\right) U+\tau_{w} V_{w}-\left(\tau_{b}+\mathcal{C}_{y y}\right) V \\
& +\nu_{t}\left[K^{p-1} \frac{\partial \tau_{y y}}{\partial y}+K \frac{\partial \tau_{y x}}{\partial x}+\tan \phi\left(\tau_{x x}-\tau_{y y}\right)\right]
\end{aligned}
$$

where,

$$
\begin{aligned}
L_{x} & =\cos \phi_{0}(\cos \phi)^{p-1}, \quad L_{y}=\left(\cos \phi_{0}\right)^{p-1}, \quad K=\cos \phi_{0} \sec \phi \\
\tau_{x x} & =K \frac{\partial U}{\partial x}, \quad \tau_{x y}=K^{p-1} \frac{\partial U}{\partial y}+K \frac{\partial V}{\partial x} \quad \text { or } \quad K \frac{\partial V}{\partial x} \\
\tau_{y y} & =K^{p-1} \frac{\partial V}{\partial y}, \quad \tau_{y x}=K^{p-1} \frac{\partial U}{\partial y}+K \frac{\partial V}{\partial x} \quad \text { or } \quad K^{p-1} \frac{\partial U}{\partial y} \\
\mathcal{C} & =\left(\begin{array}{ll}
\mathcal{C}_{x x} & \mathcal{C}_{x y} \\
\mathcal{C}_{y x} & \mathcal{C}_{y y}
\end{array}\right) \approx\left(\begin{array}{ll}
\mathcal{C}_{\lambda \lambda} & \mathcal{C}_{\lambda \phi} \\
\mathcal{C}_{\phi \lambda} & \mathcal{C}_{\phi \phi}
\end{array}\right)
\end{aligned}
$$

Finally, in order to avoid the node-to-node oscillations that arise from solving the primitive continuity equation in the CGFEM (Gray and Lynch, 1979) we reformulate it into the Generalized Wave Continuity Equation (GWCE) (Kinnmark, 1986; Westerink et al., 1992; Le Bars et al., 2010). The GWCE is obtained by differentiating the primitive continuity Eq. (S3) with 
respect to time, and adding on Eq. (S3) multiplied by a constant and positive weight, $\tau_{0}$, leading to,

$$
\begin{aligned}
\frac{\partial^{2}\left(\zeta \cos ^{p} \phi\right)}{\partial t^{2}}+\tau_{0} \frac{\partial\left(\zeta \cos ^{p} \phi\right)}{\partial t} & =-L_{x} \frac{\partial J_{x}}{\partial x}-L_{y} \frac{\partial\left(J_{y} \cos \phi\right)}{\partial y} \\
J_{x} & =H \frac{\partial U}{\partial t}+U \frac{\partial \zeta}{\partial t}+\tau_{0} U H \\
J_{y} & =H \frac{\partial V}{\partial t}+V \frac{\partial \zeta}{\partial t}+\tau_{0} V H
\end{aligned}
$$$$
30
$$

where $\frac{\partial U}{\partial t}$ and $\frac{\partial V}{\partial t}$ are found by substituting in the momentum Eqs. (S4), (S5). The final set of equations that are actually solved by ADCIRC are Eqs. (S4), (S5), and (S6).

\section{S1.2 Comparison to Previous Formulation}

The form of the primitive continuity equation in Eq. (S3), and hence the GWCE in Eq. (S6), differs from the previously employed formulation in ADCIRC by multiplying out Eq. (1) from the main manuscript by $\cos ^{p} \phi$. Doing so avoids the appearance of the following nonlinear term which Kolar et al. (1994a) expands out,

$$
\frac{1}{R \cos \phi} \frac{\partial(V H \cos \phi)}{\partial \phi}=\frac{1}{R} \frac{\partial(V H)}{\partial \phi}-\frac{\tan \phi}{R} V H
$$

Performing the above expansion removes the nonlinearity of the LHS of Eq. (S9) but the appearance of the stiff $\frac{\tan \phi}{R} V H$ term on the RHS is problematic since it approaches infinity near the poles, and its solution in the FEM using the GWCE does not lead to mass conservation. In fact, we tried solving this form of the equations and found it to be inherently unstable for simulation. Furthermore, upon inspection of the 1994 ADCIRC code related to Kolar et al. (1994a), as well as present codes, this term could not be found. Thus, we can only presume that it has always been omitted entirely in the ADCIRC code. With this omission the equations solved no longer takes into account the Earth's curvature. This may be acceptable for local or regional domains (ADCIRC has traditionally been used to simulate the Western North Atlantic regional domain), but certainly not global domains.

In contrast, the form of Eq. (S3) presented here avoids the need to expand out the LHS of Eq. (S9) because the $\frac{1}{\cos \phi}$ multiple is canceled out. It is clear that this form of the equations has desirable well-balanced properties that lead to mass conservation and stability (cf. Hervouet, 2007; Castro et al., 2018). The only potential inconvenience arises from the requirement to now solve for $\zeta \cos ^{p} \phi$ in Eq. (S3) instead of $\zeta$. However, in practice $\cos ^{p} \phi$ may be lumped in with other coefficients on the left-hand side to recover $\zeta$ directly if we set $\cos ^{p} \phi$ to be constant across an element. Note also that in the equal-area projection $(p=0)$ the $\cos ^{p} \phi$ multiple is simply equal to 1 . Moreover, in the case when $p=1$, the formulation shown here is equivalent to that used by Castro et al. (2018), and in the case when $p=2$, the formulation is equivalent to that used in TELEMAC (Hervouet, 2007).

\section{S1.3 Coordinate Rotation to Remove Pole Singularity}

55 Even though a mesh can be generated on the sphere to cover the entire Earth, the spherical coordinate system prohibits the placement of a vertex on a pole due to the appearance of a singularity, and the cylindrical mapping system adopted by the numerical model precludes any element from covering over a pole (Fig. S1). To avoid this problem, FVCOM locally switches to the stereographic projection in the vicinity of the North Pole (Chen et al., 2016). Alternatively, the governing equations can be formulated in terms of local coordinates avoiding the spherical coordinate form altogether (Comblen et al., 2009). Here, we take advantage of the current positioning of Earth's landmasses (antipodes) and rotate the $(\lambda, \phi)$ coordinates before applying the cylindrical projection so that the numerical poles are removed from the ocean domain, i.e., the poles are rotated onto land (Fig. S1). In doing so we also rotate the Coriolis vector, the surface wind vectors and the internal tide wave drag tensor in Eqs. (S4), (S5). 

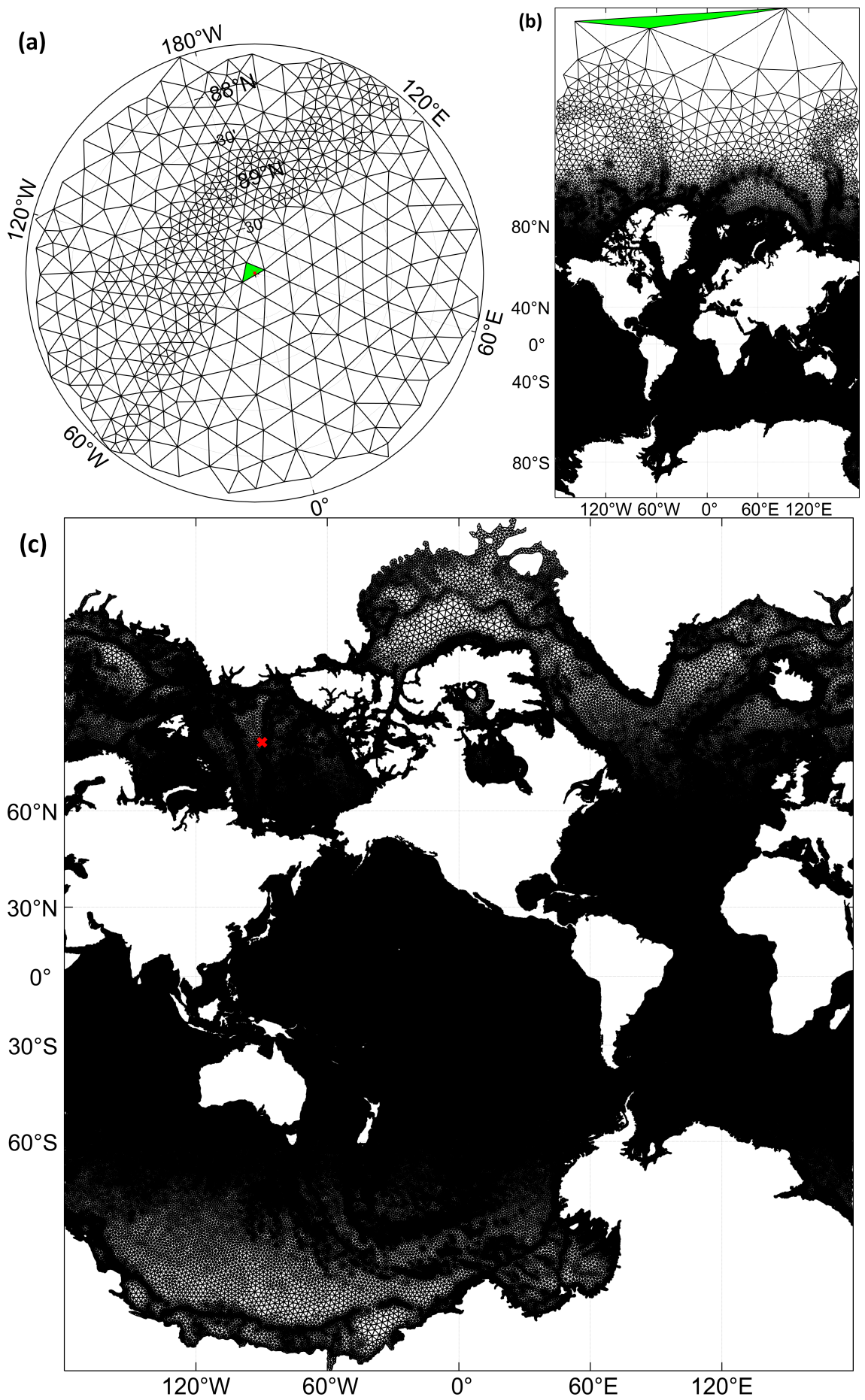

Figure S1. Triangulations of the MinEle-C mesh design (refer to the main manuscript for design details). (a) Stereographic projection zoomin to the North Pole (red dot) with the element covering the North Pole colored green; (b) Mercator projection showing that the green colored element from (a) becomes flat on the cylindrical mapping system; (c) Mercator projection of the rotated mesh that places the new North Pole in the center of Greenland; the red cross indicates the new position of the centroid of the green color element in (a). 


\section{S1.4 Numerical Solution Scheme}

65 Here we present the temporal integration solution scheme of Eqs. (S4)-(S6). Readers are referred to Luettich and Westerink (2004) for details on the CG-FEM spatial discretization. For computational efficiency the method allows for the momentum equations to be decoupled from the GWCE and solved separately in succession. First, the GWCE equations are solved for $\zeta^{* s+1} \equiv \zeta^{s+1}-\zeta^{s}$ ( $s$ indicates the time level) using a three time level approximation of $\zeta$ in the gravity wave term $\left(g H \frac{\partial \zeta}{\partial x}\right)$. The solution of the GWCE equations are of the form:

$$
\begin{aligned}
\frac{\zeta^{* s+1} \cos ^{p} \phi}{\Delta t} & {\left[\frac{1}{\Delta t}+\frac{\tau_{0}}{2}\right]+\alpha_{1} \mathrm{GW}\left(\zeta^{* s+1}\right)=\frac{\zeta^{* s} \cos ^{p} \phi}{\Delta t}\left[\frac{1}{\Delta t}-\frac{\tau_{0}}{2}\right] } \\
& -\left(\alpha_{1}+\alpha_{2}\right) \mathrm{GW}\left(\zeta^{s}\right)-\alpha_{3} \mathrm{GW}\left(\zeta^{s-1}\right)+L_{x} \frac{\partial \tilde{J}_{x}{ }^{s}}{\partial x}+L_{y} \frac{\partial\left(\tilde{J}_{y}{ }^{s} \cos \phi\right)}{\partial y} \\
\mathrm{GW}(z) & =L_{x} \frac{\partial}{\partial x}\left(g H K \frac{\partial z}{\partial x}\right)+L_{y} \frac{\partial}{\partial y}\left(g H \cos \phi K^{p-1} \frac{\partial z}{\partial y}\right)
\end{aligned}
$$

where $\zeta^{* s} \equiv \zeta^{s}-\zeta^{s-1}, \tilde{J}_{x}=J_{x}-g H \frac{\partial \zeta}{\partial x}$ and $\tilde{J}_{y}=J_{y}-g H \frac{\partial \zeta}{\partial y}$, i.e., the gravity wave (GW) term has been subtracted out from the barotropic pressure gradient (BPG) term $\left(\frac{\partial \Psi}{\partial x}\right)$. In the previous versions of ADCIRC only the linear component of the GW term (i.e., $g h \frac{\partial \zeta}{\partial x}$ ) is subtracted out from the BPG in order to keep the LHS matrix invariant to reduce computational cost. However, the matrix must be updated when wetting-drying is invoked anyway, and we find that solving the full GW term (i.e., $g H \frac{\partial \zeta}{\partial x}$ ) implicitly aids stability allowing for a larger time step to be employed. Equation (S10) is solved using a conjugate gradient iterative (CGI) solver when $\alpha_{1}>0$ and/or when using consistent mass-matrix exact integration (Tanaka et al., 2011). Alternatively, lumped mass nodal integration can be used along with setting $\alpha_{1}=0$ to avoid a matrix-inversion solve (Tanaka et al., 2011).

Second, the momentum equations are solved for $U^{s+1}$ and $V^{s+1}$ using a symmetrical two time level - Crank-Nicholson type - scheme which has been found to be free from numerical artifacts (Kinnmark, 1986),

$$
\begin{aligned}
U^{s+1}\left[\frac{1}{\Delta t}+\frac{\tau_{b}^{s}+\mathcal{C}_{x x}^{s}}{2}\right] & +V^{s+1} \frac{\mathcal{C}_{x y}^{s}-f^{\prime}}{2}=U^{s}\left[\frac{1}{\Delta t}-\frac{\tau_{b}^{s}+\mathcal{C}_{x x}^{s}}{2}\right]-V^{s} \frac{\mathcal{C}_{x y}^{s}-f^{\prime}}{2} \\
& +\frac{1}{2}\left[\left(\tau_{w} U_{w}\right)^{s+1}+\left(\tau_{w} U_{w}\right)^{s}\right]-\frac{K}{2}\left[\frac{\partial \Psi^{s+1}}{\partial x}+\frac{\partial \Psi^{s}}{\partial x}\right] \\
& -U^{s} K \frac{\partial U^{s}}{\partial x}-V^{s} K^{p-1} \frac{\partial U^{s}}{\partial y} \\
& +\nu_{t}^{s}\left[K \frac{\partial \tau_{x x}^{s}}{\partial x}+K^{p-1} \frac{\partial \tau_{x y}^{s}}{\partial y}-\tan \phi\left(\tau_{x y}^{s}+\tau_{y x}^{s}\right)\right]
\end{aligned}
$$

85

$$
\begin{aligned}
V^{s+1}\left[\frac{1}{\Delta t}+\frac{\tau_{b}^{s}+\mathcal{C}_{y y}^{s}}{2}\right] & +U^{s+1} \frac{\mathcal{C}_{y x}^{s}+f^{\prime}}{2}=V^{s}\left[\frac{1}{\Delta t}-\frac{\tau_{b}^{s}+\mathcal{C}_{y y}^{s}}{2}\right]-U^{s} \frac{\mathcal{C}_{y x}^{s}+f^{\prime}}{2} \\
& +\frac{1}{2}\left[\left(\tau_{w} V_{w}\right)^{s+1}+\left(\tau_{w} V_{w}\right)^{s}\right]-\frac{K^{p-1}}{2}\left[\frac{\partial \Psi^{s+1}}{\partial y}+\frac{\partial \Psi^{s}}{\partial y}\right] \\
& -U^{s} K \frac{\partial V^{s}}{\partial x}-V^{s} K^{p-1} \frac{\partial V^{s}}{\partial y} \\
& +\nu_{t}^{s}\left[K^{p-1} \frac{\partial \tau_{y y}^{s}}{\partial y}+K \frac{\partial \tau_{y x}^{s}}{\partial x}+\tan \phi\left(\tau_{x x}^{s}-\tau_{y y}^{s}\right)\right]
\end{aligned}
$$

Equations (S12), (S13) are solved explicitly using a lumped mass matrix (Tanaka et al., 2011). Thus, ADCIRC is able to significantly reduce the additional computational time usually associated with FEM models because it only requires one block diagonal matrix-inversion solve in the GWCE when using the semi-implicit scheme/consistent mass-matrix exact integration. 
In previous research, stability analysis of the numerical scheme has been conducted primarily on the Wave Continuity Equation (WCE) (Lynch and Gray, 1979), which is a special case of the GWCE when $\tau_{0}=\tau_{b}$. It has been shown that the WCE is thirdorder accurate and unconditionally stable with the following choice of weighting factors (Lynch and Gray, 1979; Foreman, 1983),

$\alpha_{1}=\alpha_{3}=\kappa \geq 1 / 4, \quad \alpha_{2}=1-2 \kappa$

However, the stability of the solution to the GWCE Eq. (S10) depends strongly on the selection of $\tau_{0}$ in addition to the weighting factors, $\alpha_{1,2,3}$ (Kinnmark, 1986). In fact, empirical evidence by multiple ADCIRC studies suggests that the choice of weighting factors in Eq. (S14) does not lead to an unconditionally stable scheme. Instead, the time step is bounded by the CFL restriction on the gravity wave speed $(\sqrt{g H} \Delta t / \Delta x<C r$, in which a practical upper bound of $C r$ is 0.5 or smaller (Dresback and Kolar, 2002)). It has been suggested this restriction on the stability of the GWCE is dominated by the nonlinear terms (Dresback and Kolar, 2002). Although it is true that nonlinear terms will prohibit unconditional stability, the implicit form of the GW term in Eq. (S10) and of the barotropic pressure gradient term $\left(\frac{\partial \Psi}{\partial x}\right)$ in Eqs.(S12), (S13) should remove the CFL restriction. To demonstrate this fact we conduct a von Neumann stability analysis on the one-dimensional (1-D) linear GWCE equations, detailed in Sect. S1.5.1.

Kinnmark (1986) used a von Neumann analysis to show that for the choice of weighting factors in Eq. (S14), the set of equations Eqs. (S17), (S18) are only unconditionally stable when $\tau_{0} \leq \tau_{b}$. Keep in mind that this means that $\tau_{0}$ has to be smaller than the minimum value of $\tau_{b}$ in the computational domain, which is a very strict requirement on $\tau_{0}$ in deep water (e.g., $\tau_{b} \approx 10^{-6} \mathrm{~s}^{-1}$ if $h=1000 \mathrm{~m}, C_{f}=0.01, U=0.1 \mathrm{~ms}^{-1}$ ). Moreover, this requirement is at odds with the suggestion that $\tau_{b}<\tau_{0}<10 \tau_{b}$ for good mass balance and solution properties (Kolar et al., 1994b), where $\tau_{b}$ here is the maximum value in the computational domain.

Further inspection of Kinnmark's stability equations (Sect. S1.5.1) also reveals that the scheme using the weights in Eq. (S14) is stable for any $\tau_{0}$ if $C r \leq 4 / 3$ when using exact integration. Thus, based on empirical evidence we can assume that in practice models do not satisfy $\tau_{0} \leq \tau_{b}$ everywhere, and are thus bounded by the aforementioned CFL constraint $(C r \leq 4 / 3$ in 1 -D, which is typically reduced by a $\sqrt{2} / 2$ multiple in 2-D (Kinnmark and Gray, 1984)) when using the choice of weighting factors in Eq. (S14).

To try and circumvent the $\tau_{0} \leq \tau_{b}$ stability requirement, we propose an alternative (non-centered) weighting scheme,

$\alpha_{1}=\alpha_{2}=\kappa, \quad \alpha_{3}=1-2 \kappa$

The aim of this weighting scheme is to place greater weight on the future and current time levels than the previous time level, i.e., improve the implicit nature of the scheme compared to Eq. (S14). In turns out that this scheme is unconditionally stable when using exact integration if:

$$
1 / 3 \leq \kappa \leq 1 / 2, \quad \tau_{0} \Delta t \leq \frac{16}{3}(3 \kappa-1)
$$

hence the strict requirement on $\tau_{0}\left(\leq \tau_{b}\right)$ for unconditional stability in the centered scheme has been eliminated, with $\kappa=1 / 2$ providing the most relaxed constraints for $\tau_{0} \Delta t$.

\section{S1.5.1 Stability Analysis of the 1-D Linearized GWCE}

The linearized 1-D form of Eqs. (S10)-(S13), sans atmospheric and astronomical forcing terms (hence the internal wave drag tensor is also omitted) is as follows,

$$
\begin{aligned}
& \frac{\zeta^{* s+1}}{\Delta t}\left(\frac{1}{\Delta t}+\frac{\tau_{0}}{2}\right)+\alpha_{1} g h \frac{\partial^{2} \zeta^{* s+1}}{\partial x^{2}}=\frac{\zeta^{* s}}{\Delta t}\left(\frac{1}{\Delta t}-\frac{\tau_{0}}{2}\right) \\
& -\left(\alpha_{1}+\alpha_{2}\right) g h \frac{\partial^{2} \zeta^{s}}{\partial x^{2}}-\alpha_{3} g h \frac{\partial^{2} \zeta^{s-1}}{\partial x^{2}}+\left(\tau_{0}-\tau_{b}^{s}\right) \frac{\partial U^{s}}{\partial x}
\end{aligned}
$$


$U^{s+1}\left(\frac{1}{\Delta t}+\frac{\tau_{b}^{s}}{2}\right)=U^{s}\left(\frac{1}{\Delta t}-\frac{\tau_{b}^{s}}{2}\right)-\frac{g}{2}\left(\frac{\partial \zeta^{s}}{\partial x}+\frac{\partial \zeta^{s+1}}{\partial x}\right)$

130 The last term on the right hand side of Eq. (S17) is responsible for the difference between the WCE and GWCE.

Herein a von Neumann/Fourier stability analysis of the linearized 1-D GWCE Eq. (S17) and non-conservative momentum Eq. (S18) solved using linear finite-elements in space is conducted following Kinnmark (1986). In this analysis the water depth, $h$, is assumed to be constant and mesh vertices are assumed to be equally spaced. Selecting a solution of the form $e^{i(\beta \Delta t+\sigma j \Delta t)}$ yields the following third-order polynomial equation for the propagation factor $\lambda=e^{i \beta \Delta t}$,

$135 a_{0}+a_{1} \lambda+a_{2} \lambda^{2} a_{3} \lambda^{3}=0$

$a_{3}=\left(1+T_{0}+4 F \alpha_{1}\right)\left(1+T_{b}\right)$

$a_{2}=\left(1+T_{0}+4 F \alpha_{1}\right)\left(-1+T_{b}\right)+\left(-2+4 F \alpha_{2}\right)\left(1+T_{b}\right)+\left(4 E / A^{2}\right)\left(T_{0}-T_{b}\right)$

$a_{1}=\left(1-T_{0}+4 F \alpha_{3}\right)\left(1+T_{b}\right)+\left(-2+4 F \alpha_{2}\right)\left(-1+T_{b}\right)+\left(4 E / A^{2}\right)\left(T_{0}-T_{b}\right)$

$a_{0}=\left(1-T_{0}+4 F \alpha_{3}\right)\left(-1+T_{b}\right)$

140 where,

$T_{0}=\tau_{0} \Delta t / 2$

$T_{b}=\tau_{b} \Delta t / 2$

$A=1-m \sin ^{2}(\sigma \Delta x / 2)$

$m= \begin{cases}2 / 3 & \text { for consistent mass-matrix exact integration } \\ 0 & \text { for lumped mass-matrix nodal integration }\end{cases}$

145

$F=\frac{C r^{2}}{A} \sin ^{2}(\sigma \Delta x / 2)$

$E=A F \cos ^{2}(\sigma \Delta x / 2)$

and we define the following,

$$
\begin{aligned}
p_{0} & =a_{3}-a_{2}+a_{1}-a_{0} \\
p_{1} & =3 a_{3}-a_{2}-a_{1}+3 a_{0} \\
150 & p_{2}=3 a_{3}+a_{2}-a_{1}-3 a_{0} \\
p_{3} & =a_{3}+a_{2}+a_{1}+a_{0} \\
\Delta_{2} & =p_{1} p_{2}-p_{0} p_{3}
\end{aligned}
$$

A necessary and sufficient condition for stability, arising from Routh-Hurwitz criterion, requires that $p_{0,1,2,3}>0$ and $\Delta_{2}>0$ (Kinnmark, 1986).

To simplify the equations we introduce a weighting scheme that reduces $\alpha_{1,2,3}$ to a single variable, $\kappa$. We investigate a centered scheme followed by a non-centered scheme skewed towards the newest time levels. 


\section{S1.5.2 Centered scheme}

First, the following centered scheme is chosen: $\alpha_{1}=\alpha_{3}=\kappa$, and $\alpha_{2}=1-2 \kappa$. This reduces $p_{0,1,2,3}, \Delta_{2}$ to the following,

$$
\begin{aligned}
p_{0} & =8[1+F(4 \kappa-1)] \\
p_{1} & =8\left[T_{0}+T_{b}+F T_{b}(4 \kappa-1)-\left(E / A^{2}\right)\left(T_{0}-T_{b}\right)\right] \\
p_{2} & =8\left[F+T_{0} T_{b}\right] \\
p_{3} & =8\left[F T_{b}+\left(E / A^{2}\right)\left(T_{0}-T_{b}\right)\right] \\
\Delta_{2} & =64\left[F T_{0}+T_{0} T_{b}^{2}+T_{0}^{2} T_{b}+F T_{0} T_{b}^{2}(4 \kappa-1)-\left(E / A^{2}\right)\left(T_{0}-T_{b}\right)\left(1+4 F \kappa+T_{0} T_{b}\right)\right]
\end{aligned}
$$

Which leads to the following conditions for stability,

\begin{tabular}{c|c|c} 
Equation & $C r \rightarrow \infty$ & $\tau_{0} \rightarrow \infty$ \\
\hline$p_{0}$ & $\kappa \geq 1 / 4$ & $\kappa<1 / 4$ and $C r^{2} \leq \frac{1-m}{1-4 \kappa}$ \\
$p_{1}$ & $\kappa \geq 1 / 4$ and $\tau_{0} \leq 4 \kappa \tau_{b}$ & $C r^{2} \leq 4(m / 2-1)^{2}$ \\
$p_{2}$ & none & none \\
$p_{3}$ & none & none \\
$\Delta_{2}$ & $\kappa \geq 1 / 4$ and $\tau_{0} \leq \tau_{b}$ & $C r^{2} \leq 4(m / 2-1)^{2}$
\end{tabular}

Thus, for any $C r$, the centered scheme is stable if $\kappa \geq 1 / 4$ and $\tau_{0} \leq \tau_{b}$. Alternatively, it is stable if $\kappa \geq 1 / 4$ and $C r^{2} \leq$ $4(m / 2-1)^{2}$, or $\kappa<1 / 4$ and $C r^{2} \leq \frac{1-m}{1-4 \kappa}$. The stability constraints are similar to those for the WCE presented by Lynch and Gray (1979), sans the requirement on $\tau_{0}$.

\section{S1.5.3 Non-centered scheme}

170 Second, a non-centered scheme skewed towards the $s+1$ and $s$ time levels is chosen: $\alpha_{1}=\alpha_{2}=\kappa$, and $\alpha_{3}=1-2 \kappa$. This reduces $p_{0,1,2,3}, \Delta_{2}$ to the following,

$$
\begin{aligned}
p_{0} & =8[1+F(1-2 \kappa)] \\
p_{1} & =8\left[T_{0}+T_{b}+F\left(6 \kappa-2+T_{b}(1-2 \kappa)\right)-\left(E / A^{2}\right)\left(T_{0}-T_{b}\right)\right] \\
p_{2} & =8\left[T_{0} T_{b}+F\left(1+2 T_{b}(3 \kappa-1)\right)\right] \\
p_{3} & =8\left[F T_{b}+\left(E / A^{2}\right)\left(T_{0}-T_{b}\right)\right] \\
\Delta_{2} & =64\left[2 F^{2}\left(T_{b}^{2}\left(-6 \kappa^{2}+\kappa\left(5-T_{0}\right)-1\right)+2 T_{b}\left(9 \kappa^{2}-6 \kappa+1\right)+3 \kappa\right)\right. \\
& \left.+F\left(T_{0} T_{b}^{2}+12 T_{0} T_{b} \kappa-4 T_{0} T_{b}+T_{0}+6 T_{b}^{2} \kappa-2 T_{b}^{2}\right)+F T_{0}+T_{0}^{2} T_{b}+T_{0} T_{b}^{2}\right]
\end{aligned}
$$$$
175
$$

Which leads to the following conditions for stability,

\begin{tabular}{c|c|c} 
Equation & $C r \rightarrow \infty$ & $\tau_{0} \rightarrow \infty$ \\
\hline$p_{0}$ & $\kappa \leq 1 / 2$ & $\kappa>1 / 2$ and $C r^{2} \leq \frac{1-m}{2 \kappa-1}$ \\
$p_{1}$ & $1 / 3 \leq \kappa \leq 1 / 2$ and $\tau_{0} \Delta t \leq 4(2-m)(3 \kappa-1)$ & $C r^{2} \leq 4(m / 2-1)^{2}$ \\
$p_{2}$ & $\kappa \geq 1 / 3$ & none \\
$p_{3}$ & none & none \\
$\Delta_{2}$ & $\kappa \geq 1 / 3$ & none
\end{tabular}

Thus, for any $C r$, the non-centered scheme is stable if $1 / 3 \leq \kappa \leq 1 / 2$ and $\tau_{0} \Delta t \leq 4(2-m)(3 \kappa-1)$. Alternatively, it is stable if $1 / 3 \leq \kappa \leq 1 / 2$ and $C r^{2} \leq 4(m / 2-1)^{2}$, or $\kappa>1 / 2$ and $C r^{2} \leq \frac{1-m}{2 \kappa-1}$.

\section{S2 Model Specifications}

In the following sections we detail the pertinent model specifications for the ADCIRC v55 code used in this study. The resulting model setup containing the mesh and input files has been archived in Pringle (2020). 
1. The momentum equations are used in non-conservative form with the lateral stress tensor in a symmetrical velocitybased form as written in Eqs. (2), (3) of the main manuscript. The full gravity wave term is solved implicitly in the GWCE as written in Eqs. (S10), (S11). In ADCIRC, the aforementioned corresponds to setting the 'fort.15' control file parameter, IM, to a value of 513113 (see: https://wiki.adcirc.org/wiki/IM).

2. The Mercator projection ( $p=2$ in Eq. (S2)) is adopted because of its conformal property. To enable this in an ADCIRC simulation the 'fort.15' control file parameter, ICS, is set to a value of 22 (see: https://wiki.adcirc.org/wiki/ICS).

3. The mesh is rotated internally within ADCIRC to remove the pole singularity (Sect. S1.3). To enable this in an ADCIRC simulation the 'fort.15' control file parameter, ICS, is set to a negative value (i.e, ICS = -22, see: https://wiki.adcirc.org/ wiki/ICS). In addition, a 'fort.rotm' input file is provided that indicates the desired rotation (we choose the "GreenlandAntarctica" option listed at https://wiki.adcirc.org/wiki/Fort.rotm).

4. $\kappa=0.5$ in Eq. (S15) is adopted because it leads to the least restrictive condition on $\tau_{0} \Delta t$ in Eq. (S16). The corresponding 'fort.15' control file weighting factors, A00, B00, C00, are set to 0.5, 0.5, 0 (see: https://wiki.adcirc.org/wiki/A00,_B00, C00).

5. $\Delta t$ is set to approximately the largest value that enables reliably stable simulations based on experience and trial-anderror. Although the linear CFL condition is satisfied unconditionally, nonlinear terms introduce instabilities on finer meshes in shallow depths, and could be related to the CFL condition based on the fluid velocity (instead of the gravity wave speed), i.e., $\mathrm{Cr}=U_{\max } \Delta t / \Delta x . \Delta t=120 \mathrm{~s}$ was used for all simulations on the global mesh without local refinement, while the stable $\Delta t$ was generally smaller for the storm tide simulations on the meshes with local refinement. Hurricane Katrina: $\Delta t=120 \mathrm{~s}$ on the MinEle $=500-\mathrm{m}$ mesh, and $\Delta t=50 \mathrm{~s}$ on the MinEle $=150-\mathrm{m}$ mesh. Super Typhoon Haiyan: $\Delta t=80 \mathrm{~s}$ on the MinEle $=500-\mathrm{m}$ mesh, and $\Delta t=30 \mathrm{~s}$ on the MinEle $=150-\mathrm{m} \mathrm{mesh}$. Based on these results and rearranging the CFL condition for the maximum fluid velocity, $U_{\max }$ with $\mathrm{Cr}$ set to 1 as the stability criteria and using the actual minimum element edgelengths of the mesh (rather than the nominal minimum resolution, MinEle) we obtain $U_{\max }=1.1-2.5 \mathrm{~m} / \mathrm{s}$. Therefore, setting $U_{\max }$ to $2.5 \mathrm{~m} / \mathrm{s}$ in the fluid velocity-based CFL condition could be used as a guideline for determining a stable $\Delta t$ for ADCIRC simulations using the semi-implicit time integration. However, this is only a guideline and does not guarantee stability. The corresponding ADCIRC 'fort.15' control file parameter for $\Delta t$ is DTDP (https://wiki.adcirc.org/wiki/DTDP).

6. We set $\tau_{0}=8 /(5 \Delta t)$, which is chosen to satisfy the stability criteria for $\tau_{0} \Delta t$ in Eq. (S16), with $\kappa=0.5$ and a safety factor of 0.6 applied to account for any possible 2-D effects. For instance, if $\Delta t=120 \mathrm{~s}$ then $\tau_{0}=1 / 75 \mathrm{~s}^{-1}$. The corresponding ADCIRC 'fort.15' control file parameter for $\tau_{0}$ is TAU0 (https://wiki.adcirc.org/wiki/TAU0).

7. Wetting-drying is enabled but its action is limited to regions with very large tidal ranges or the storm landfall regions because the meshes were built without an overland floodplain in this study. To enable wetting-drying in an ADCIRC simulation, the 'fort.15' control file parameter, NOLIFA, is set to 2 (see: https://wiki.adcirc.org/wiki/NOLIFA).

\section{S2.2 Bathymetric Interpolation}

To interpolate the bathymetry from the Digital-Elevation-Model (DEM) structured grid to the unstructured mesh vertices we use a cell-averaging technique native to the OceanMesh2D software, called through the "interp" function wrapper (cf. Roberts et al., 2019). Given the resolution of the meshes and accuracy of the DEM data used in this study, a $5 \mathrm{~m}$ floor on the ocean depth was applied.

\section{S2.3 Tidal Potential}

The equilibrium tidal potential is prescribed internally in ADCIRC using the analytical formulation presented in Luettich and Westerink (1992, Eq. (27), p. 17). The time-dependent nodal factors and equilibrium arguments for each tidal constituent are 
computed when constructing the ADCIRC 'fort.15' control file with the "Make_f15" OceanMesh2D function wrapper based on the start and end datetimes of the simulation. Specifically, the nodal factor is set to a constant for each simulation based on the mean datetime, and the equilibrium argument is based on the start datetime. The OceanMesh2D function to compute the nodal factors and equilibrium arguments is adopted from the UTide MATLAB toolbox (Codiga, 2011). In addition, the ADCIRC 'fort.15' control file parameter, NTIP, is set to 1 or 2 (see: https://wiki.adcirc.org/wiki/NTIP), and NTIF is set to the number of tidal constituents used (https://wiki.adcirc.org/wiki/NTIF) - this is automatically handled by the "Make_f15" function.

\section{S2.4 Self-attraction and Loading Tide}

We prescribe the self-attraction and loading (SAL) tide by reconstructing the elevation signal from harmonic constituents provided by the FES2014 (Lyard et al., 2006) data assimilated tidal solutions (ftp://ftp.legos.obs-mip.fr/pub/FES2012-project/ data/LSA/FES2014/). These are linearly interpolated from the FES2014 structured grid onto our unstructured mesh vertices. The "Make_f24" OceanMesh2D function is used to perform this process and write out the data into a 'fort.24' ADCIRC input file (see: https://wiki.adcirc.org/wiki/Fort.24_file). To use the SAL information in an ADCIRC simulation, the 'fort.15' control file parameter, NTIP, is set to a value of 2 (see: https://wiki.adcirc.org/wiki/NTIP) - this is automatically handled by the "Make_f24" function.

\section{S2.5 Atmospheric Forcing}

In this study atmospheric forcing is either, interpolated from gridded meteorological fields, or reconstructed from the symmetric Holland parametric vortex model internally in ADCIRC during the simulation. The choice of atmospheric forcing type is dictated by the ADCIRC 'fort.15' control file parameter, NWS (see: https://wiki.adcirc.org/wiki/NWS).

245 To use a single dataset of gridded meteorological fields in GRIB2 file format (e.g., CFSR and CFSv2 as used in this study), NWS is set to 14. To insert a local inset of gridded OceanWeather Inc. (OWI) ASCII file format meteorological fields into the GRIB2 file meteorology, NWS is set to -14. Simultaneously, the WTIMINC 'fort.15' control file parameter is set to the time interval of the meteorological data in seconds (if NWS $=-14$, first value is for the GRIB2 meteorology, second value is for the OWI meteorology, see: https://wiki.adcirc.org/wiki/WTIMINC).

250 To use the symmetric Holland parametric vortex model, NWS is set to 8, a 'fort.22' meteorology control file is supplied (see: https://wiki.adcirc.org/wiki/Fort.22_file_format\#NWS_.3D_8), and we choose the 'fort.15' control file parameter, BLAdj, to be 0.78 (see: https://wiki.adcirc.org/wiki/YYYY_MM_DD_HH24_StormNumber_BLAdj).

\section{S2.6 Internal Tide Wave Drag}

The "Calc_IT_Fric" OceanMesh2D function is used to compute the internal tide wave drag tensor, $\mathcal{C}$ offline using the localgeneration formulation, incorporating saturation at supercritical topography, and a cutoff depth of $250 \mathrm{~m}$ below which $\mathcal{C}$ is set to zero (cf. Pringle et al., 2018b). Critical to this computation is the determination of the topographic gradients. To perform this calculation the OceanMesh2D "interp" function wrapper is used prior to the "Calc_IT_Fric" function call. "interp" employs a cell-averaging type technique where the magnitude of the topographic gradients at the mesh vertices are computed as the root-mean-square of the topographic gradients directly computed on the DEM points located within a region determined by a measure of the local mesh size. The sign of the topographic gradient is then determined by the sign of the gradient of the interpolated bathymetry on the mesh vertices. The aim of this interpolation strategy is to ensure that sub-grid information of the topographic gradient on the original DEM is preserved. In this way the form of the internal tide wave drag tensor used here can be thought of as a hybrid of the Jayne and St. Laurent (2001) sub-grid roughness method and Lyard et al. (2006); Zaron and Egbert (2006) gradient-based methods.

265 The internal tide wave drag tensor contains a free parameter $C_{i t}$ (see the main manuscript). In this study we consider $C_{i t}$ to be a globally constant calibration coefficient that should be tuned so that the model simulation has the correct total barotropic tidal energy in the deep ocean $(h>1 \mathrm{~km})$. For this purpose we use the total available tidal potential energy of the 5-constituent 
Table 1. Calibrated $C_{i t}$ values used for each mesh design (refer to Table 2 from the main manuscript for design details).

\begin{tabular}{l|c|c|c|c} 
& \multicolumn{4}{|c}{ Design Code } \\
Variable Mesh Size Parameter & Ref & A & B & C \\
\hline MinEle & 2.13 & 2.10 & 2.02 & 1.87 \\
TLS & 2.13 & 2.01 & 1.94 & 1.65 \\
FL & 2.13 & 2.04 & 1.95 & 1.90
\end{tabular}

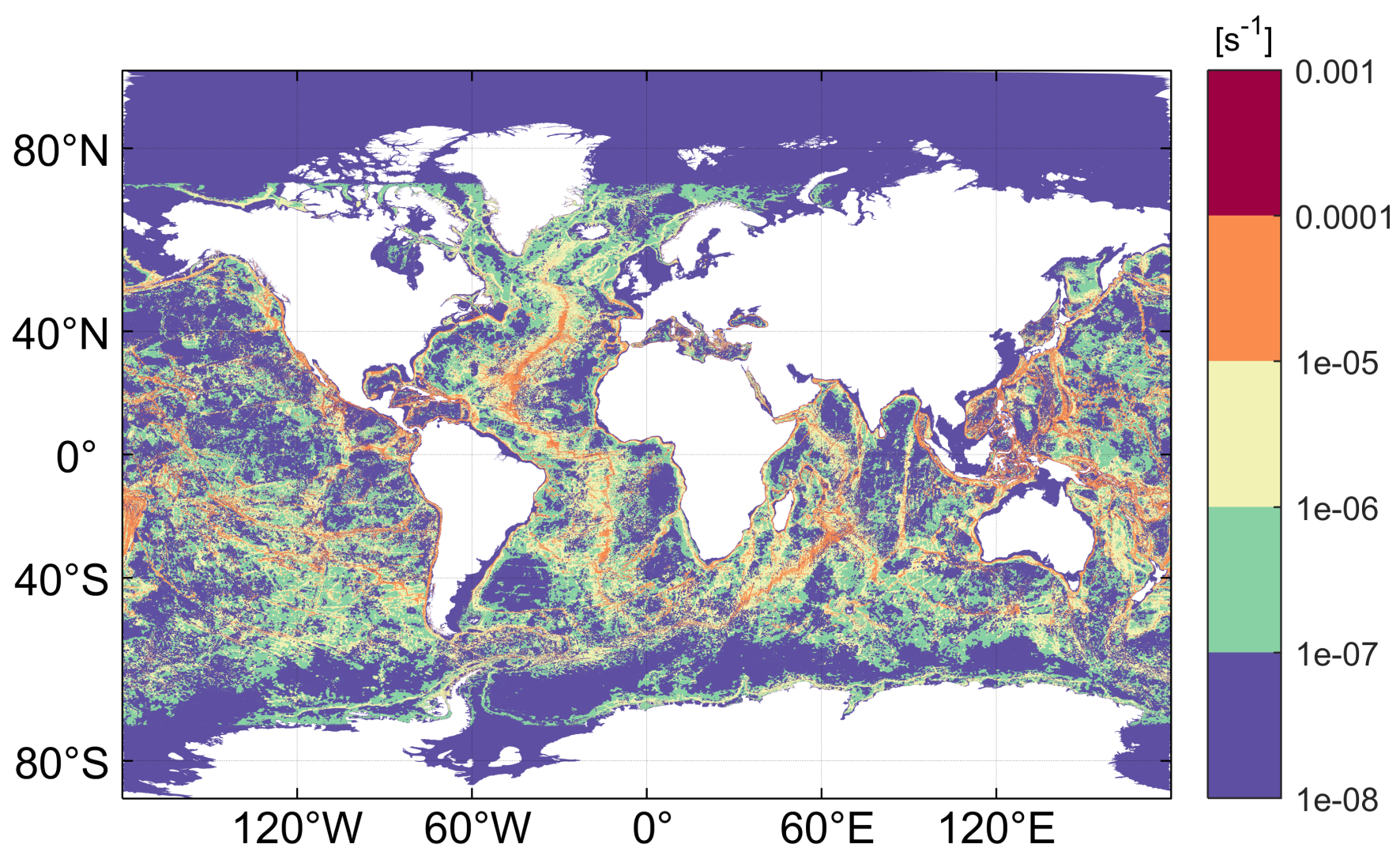

Figure S2. Map of the infinity-norm of the internal tide wave drag tensor, $\mathcal{C}$ on the reference mesh.

tidal signal,

$\mathrm{APE}_{t \mid t o t}=\frac{\rho g}{4} \iint \sum_{k=1}^{5} A_{k}^{2} d A$

270 where $A$ is the tidal amplitude, and $k$ indicates the arbitrary constituent number (amongst the five leading constituents, $\mathbf{M}_{2}, \mathrm{~S}_{2}$, $\left.\mathrm{N}_{2}, \mathrm{~K}_{1}, \mathrm{O}_{1}\right) . C_{i t}$ is varied (to a precision of \pm 0.01 ) until the simulated $\mathrm{APE}_{t \mid t o t}$ in the deep ocean matches that of the TPXO9Atlas (= $153 \mathrm{PJ}$ ). The resulting $C_{i t}$ values for the various mesh designs are shown in Table 1. A map of the infinity-norm of the $\mathcal{C}$ tensor on the reference mesh is shown in Fig. S2.

To use the internal tide wave drag tensor in an ADCIRC simulation, the data is written out as an "internal_tide_friction" 'fort.13' input file attribute (see: https://wiki.adcirc.org/wiki/Fort.13_file\#Internal_Tide_Energy_Conversion). 


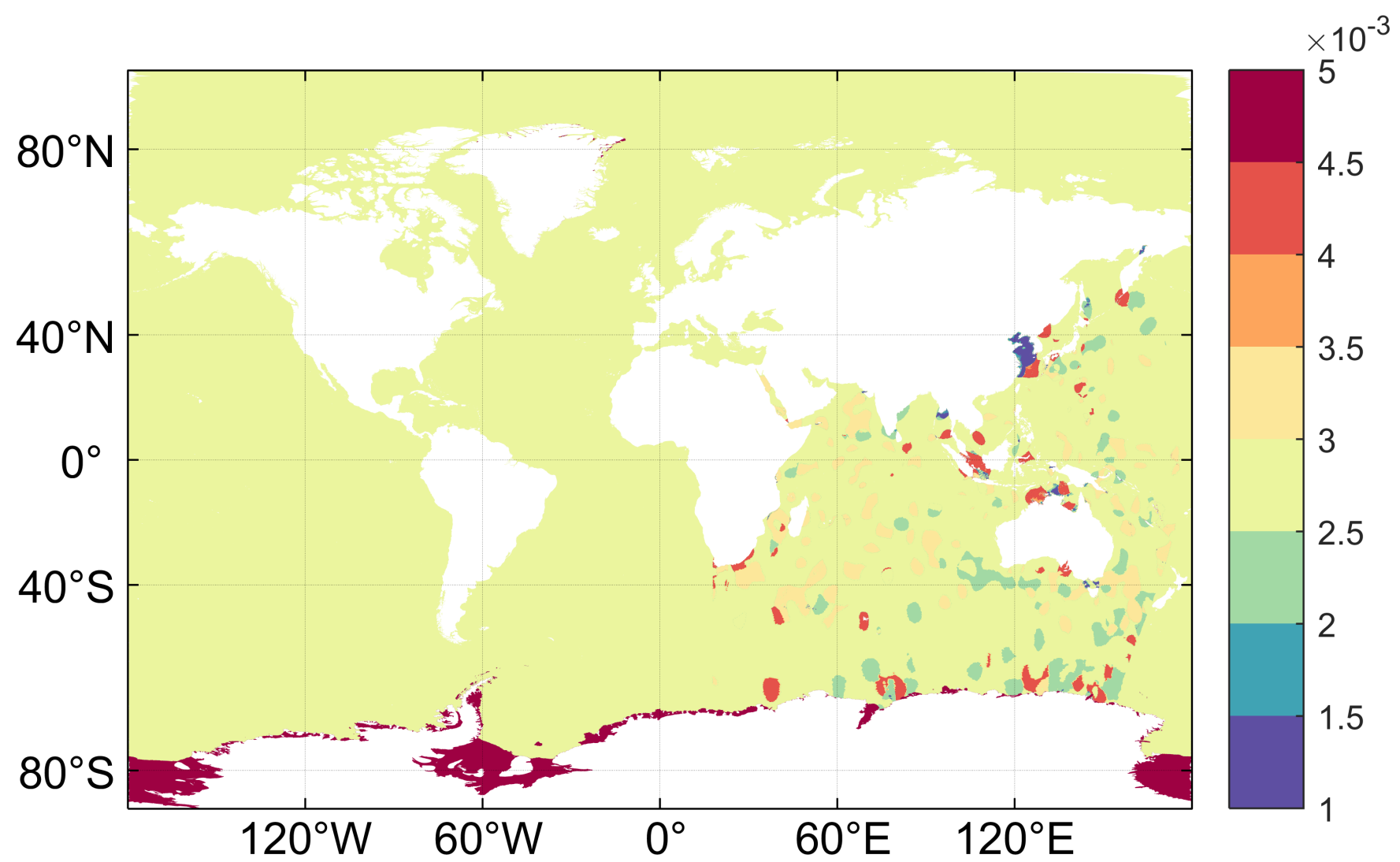

Figure S3. Map of spatially varying bottom friction coefficients, $C_{f}$ on the reference mesh.

\section{S2.7 Quadratic Bottom Friction}

For all mesh designs, $C_{f}$ in $\tau_{b}$ (refer to main manuscript) is set to 0.0025 everywhere except under the Antarctic and Greenland ice shelves where $C_{f}$ is doubled (c.f. Zaron, 2019), and in the Indian and Western Pacific Oceans where values from Pringle et al. (2018a) are used (Fig. S3). In particular, small values of $C_{f}$ (ranging between 0.00075 and 0.002) in the Yellow Sea are important to reduce the tidal error here (Lefevre et al., 2000; Pringle et al., 2018a). Other variations of $C_{f}$ outside of these regions would likely also further reduce the tidal error but the determination of a suitable unified framework to calibrate spatially varying $C_{f}$ globally is left for future work.

To use quadratic bottom friction in an ADCIRC simulation, the NOLIBF 'fort.15' control file attribute is set to 1 (see: https: //wiki.adcirc.org/wiki/NOLIBF), and the "quadratic_friction_coefficient_at_sea_floor" 'fort.13' input file attribute is used to specify spatially varying $C_{f}$ (see: https://wiki.adcirc.org/wiki/Fort.13_file\#Quadratic_Friction_coefficient).

\section{S2.8 Lateral Mixing}

The lateral mixing coefficient, $\nu_{t}$ is calculated through the Smagorinsky turbulence closure model with a coefficient of 0.2 (Dresback et al., 2005). Model results were insensitive to this coefficient for the values we tested $(0.05,0.10,0.20)$ but it can help for model stability to use a larger value.

290 To use the Smagorinksy model in an ADCIRC simulation, the ESLM 'fort.15' control file attribute is set equal to the negative value of the Smagorinksy coefficient (i.e., ESLM = -0.2) (see: https://wiki.adcirc.org/wiki/ESLM). 


\section{References}

Castro, M. J., Ortega, S., and Parés, C.: Reprint of: Well-balanced methods for the shallow water equations in spherical coordinates, Computers and Fluids, 169, 129-140, https://doi.org/10.1016/j.compfluid.2018.03.052, https://doi.org/10.1016/j.compfluid.2017.08.035, 2018.

Chen, C., Gao, G., Zhang, Y., Beardsley, R. C., Lai, Z., Qi, J., and Lin, H.: Circulation in the Arctic Ocean: Results from a high-resolution coupled ice-sea nested Global-FVCOM and Arctic-FVCOM system, Progress in Oceanography, 141, 60-80, https://doi.org/10.1016/j.pocean.2015.12.002, http://dx.doi.org/10.1016/j.pocean.2015.12.002, 2016.

Codiga, D. L.: Unified Tidal Analysis and Prediction Using the UTide Matlab Functions, Tech. Rep. 01, Graduate School of Oceanography, University of Rhode Island, Narragansett, RI, https://doi.org/10.13140/RG.2.1.3761.2008, 2011.

Comblen, R., Legrand, S., Deleersnijder, E., and Legat, V.: A finite element method for solving the shallow water equations on the sphere, Ocean Modelling, 28, 12-23, https://doi.org/10.1016/j.ocemod.2008.05.004, http://dx.doi.org/10.1016/j.ocemod.2008.05.004, 2009.

Dresback, K. M. and Kolar, R. L.: An implicit time-marching algorithm for shallow water models based on the generalized wave continuity equation, International Journal for Numerical Methods in Fluids, 36, 925-945, https://doi.org/10.1002/fld.157.abs, 2002.

Dresback, K. M., Kolar, R. L., and Dietrich, J. C.: On the form of the momentum equation for shallow water models based on the generalized wave continuity equation, Advances in Water Resources, 28, 345-358, https://doi.org/10.1016/j.advwatres.2004.11.011, 2005.

Foreman, M. G. G.: An Anaysis of the "Wave Equation" Model for Finite Element Tidal Computations, Journal of Computational Physics, 52, 290-312, 1983.

Gray, W. G. and Lynch, D. R.: On the control of noise in finite element tidal computations: A semi-implicit approach, Computers and Fluids, 7, 47-67, https://doi.org/10.1016/0045-7930(79)90005-7, 1979.

Hervouet, J.-M.: Equations of free surface hydrodynamics, in: Hydrodynamics of Free Surface Flows: Modelling with the Finite Element Method, chap. 2, pp. 5-75, John Wiley \& Sons, Ltd, https://doi.org/10.1002/9780470319628.ch2, https://onlinelibrary.wiley.com/doi/abs/ 10.1002/9780470319628.ch2, 2007.

Jayne, S. R. and St. Laurent, L. C.: Parameterizing tidal dissipation over rough topography, Geophysical Research Letters, 28, 811-814, https://doi.org/10.1029/2000GL012044, http://doi.wiley.com/10.1029/2000GL012044, 2001.

Kinnmark, I. P.: The Shallow Water Wave Equations: Formulation, Analysis and Application, vol. 15 of Lecture Notes in Engineering, Springer Berlin Heidelberg, Berlin, Heidelberg, https://doi.org/10.1007/978-3-642-82646-7, http://link.springer.com/10.1007/ 978-3-642-82646-7, 1986.

Kinnmark, I. P. and Gray, W. G.: A Two-Dimensional Analysis of the Wave Equation Model for Finite Element Tidal Computations, International Journal for Numerical Methods in Engineering, 20, 369-383, 1984.

Kolar, R. L., Gray, W. G., Westerink, J. J., and Luettich, R. A.: Shallow water modeling in spherical coordinates: Equation formulation, numerical implementation, and application: Modélisation des équations de saint-venant, en coordonnées sphériques: Formulation, resolution numérique et application, Journal of Hydraulic Research, 32, 3-24, https://doi.org/10.1080/00221689409498786, 1994a.

Kolar, R. L., Westerink, J. J., Cantekin, M. E., and Blain, C. A.: Aspects of Nonlinear Simulations using Shallow-water Models based on the Wave Continuity Equation, Computers \& Fluids, 23, 523-538, 1994b.

Le Bars, Y., Lyard, F., Jeandel, C., and Dardengo, L.: The AMANDES tidal model for the Amazon estuary and shelf, Ocean Modelling, 31, 132-149, https://doi.org/10.1016/j.ocemod.2009.11.001, http://dx.doi.org/10.1016/j.ocemod.2009.11.001, 2010.

Lefevre, F., Provost, C. L., and Lyard, F. H.: How can we improve a global ocean tide model at a region scale? A test on the Yellow Sea and the East China Sea, Journal of Geophysical Research: Oceans, 105, 8707-8725, https://doi.org/10.1029/1999JC900281, 2000.

Luettich, R. A. and Westerink, J. J.: ADCIRC: an advanced three-dimensional circulation model for shelves coasts and estuaries, report 1: theory and methodology of ADCIRC-2DDI and ADCIRC-3DL, Dredging Research Program, Tech. rep., DRP-92-6, U.S. Army Engineers Waterways Experiment Station, Vicksburg, MS, 1992.

Luettich, R. A. and Westerink, J. J.: Formulation and Numerical Implementation of the 2D/3D ADCIRC Finite Element Model Version 44.XX, Tech. rep., University of North Carolina at Chapel Hill \& University of Notre Dame, 2004.

Lyard, F., Lefevre, F., Letellier, T., and Francis, O.: Modelling the global ocean tides: modern insights from FES2004, Ocean Dynamics, 56, 394-415, https://doi.org/10.1007/s10236-006-0086-x, http://link.springer.com/10.1007/s10236-006-0086-x, 2006.

Lynch, D. R. and Gray, W. G.: A Wave Equation Model for Finite Element Tidal Computations, Computers \& Fluids, 7, 207-228, 1979.

Pringle, W. J.: Global Storm Tide Modeling on Unstructured Meshes with ADCIRC v55 - Simulation Results and Model Setup, https://doi.org/10.5281/zenodo.3911282, 2020.

Pringle, W. J., Wirasaet, D., Suhardjo, A., Meixner, J., Westerink, J. J., Kennedy, A. B., and Nong, S.: Finite-Element Barotropic Model for the Indian and Western Pacific Oceans: Tidal Model-Data Comparisons and Sensitivities, Ocean Modelling, 129, 13-38, https://doi.org/10.1016/j.ocemod.2018.07.003, https://linkinghub.elsevier.com/retrieve/pii/S146350031830026X, 2018a.

Pringle, W. J., Wirasaet, D., and Westerink, J. J.: Modifications to Internal Tide Conversion Parameterizations and Implementation into Barotropic Ocean Models, EarthArXiv, p. 9, https://doi.org/10.31223/osf.io/84w53, 2018b. 
Roberts, K. J., Pringle, W. J., and Westerink, J. J.: OceanMesh2D 1.0: MATLAB-based software for two-dimensional unstructured mesh generation in coastal ocean modeling, Geoscientific Model Development, 12, 1847-1868, https://doi.org/10.5194/gmd-12-1847-2019, 2019.

Tanaka, S., Bunya, S., Westerink, J. J., Dawson, C., and Luettich, R. A.: Scalability of an Unstructured Grid Continuous Galerkin Based Hurricane Storm Surge Model, Journal of Scientific Computing, 46, 329-358, https://doi.org/10.1007/s10915-010-9402-1, http://link. springer.com/10.1007/s10915-010-9402-1, 2011.

350 Westerink, J. J., Luettich, R. A., Baptists, A. M., Scheffner, N. W., and Farrar, P.: Tide and Storm Surge Predictions Using Finite Element Model, Journal of Hydraulic Engineering, 118, 1373-1390, https://doi.org/10.1061/(ASCE)0733-9429(1992)118:10(1373), http: //ascelibrary.org/doi/10.1061/\{\%\}28ASCE\{\%\}290733-9429\{\%\}281992\{\%\}29118\{\%\}3A10\{\%\}281373\{\%\}29, 1992.

Zaron, E. D.: Simultaneous Estimation of Ocean Tides and Underwater Topography in the Weddell Sea, Journal of Geophysical Research: Oceans, 124, 3125-3148, https://doi.org/10.1029/2019JC015037, 2019.

355 Zaron, E. D. and Egbert, G. D.: Estimating Open-Ocean Barotropic Tidal Dissipation: The Hawaiian Ridge, Journal of Physical Oceanography, 36, 1019-1035, https://doi.org/10.1175/JPO2878.1, http://journals.ametsoc.org/doi/abs/10.1175/JPO2878.1, 2006. 